\title{
Existence and stability of chimera states in a minimal system of phase oscillators
}

\author{
Mary Thoubaan and Peter Ashwin \\ Centre for Systems, Dynamics and Control, \\ Department of Mathematics, \\ University of Exeter, Exeter EX4 4QF, UK.
}

(Dated: August 19, 2018)

\begin{abstract}
We examine partial frequency locked weak chimera states in a network of six identical and indistinguishable phase oscillators with neighbour and next-neighbour coupling and two harmonic coupling of the form $g(\phi)=-\sin (\phi-\alpha)+r \sin 2 \phi$. We limit to a specific partial cluster subspace, reduce to a two dimensional system in terms of phase differences and show that this has an integral of motion for $\alpha=\pi / 2$ and $r=0$. By careful analysis of the phase space we show there is a continuum of neutrally stable weak chimera states in this case. We approximate the Poincaré return map for these weak chimera solutions and demonstrate several results about the stability and bifurcation of weak chimeras for small $\beta=\pi / 2-\alpha$ and $r$ that agree with numerical path following of the solutions.
\end{abstract}

Patterns of coherence and incoherence appear spontaneously due to self-organization in systems of coupled oscillators. For a particular class of solutions called chimera states, the coherence and incoherence can appear at the same time in different parts of the pattern. These patterns have inspired a lot of work since Kuramoto and Battogtokh found coupled oscillator systems with non-local coupling that reorganize themselves into such state [16]. These states were named chimeras in [2] and since then they been found numerically in a wide variety of coupled systems and in applications to a wide variety of areas, from chemical oscillations to brain dynamics. In an attempt to characterise such states more precisely, the second author and Burylko [4] characterised weak chimera states more generally as a form of partial frequency desynchronization. Also, they presented a minimal system of six oscillators that, from numerical simulations, seem to have nontrivial weak chimeras. In this paper we re-investigate this system and rigorously prove existence of weak chimeras and a nontrivial integrable case. We also show some stability results and bifurcations of weak chimeras near this integrable limit, and find good agreement with numerical path-following.

\section{INTRODUCTION}

Dynamical states where coherence (synchrony) and incoherence (asynchrony) occur at the same time have recently been studied under the name of chimera states. Such mixed states have been found in an increasingly wide range of nonlinear systems $[9,15,18,31,32]$ since their original discovery in coupled phase oscillators [16] and being named "chimeras" by Abrams and Strogatz [3]. Non-local interactions were originally regarded as essential for chimeras to emerge [11, 25]. However, chimera states have been found for systems with global coupling [24, 28-30], mean-field coupling [6] and even local cou- pling $[7,19]$. Wolfrum and Omelchenko examined the detailed dynamical properties of chimera states in small populations of coupled phase oscillators [34]. However, exact solutions for chimera state have, in general, only been found at a population level [1].

Chimeras have been studied for a wide range of system sizes $[16,35]$ and they play an important role that help to understand many complex behaviour systems in the real world. There are a wide range of applications including to chemical oscillations [17,33], electronic circuits [10, 29], mechanical oscillators [23], brain dynamics [20] and optical experiments [12].

Whether a particular system state is a chimera depends critically on the definition of coherence and incoherence. A particular approach was introduced by Ashwin and Burylko [4] who define a weak chimera as a type of invariant set with partial frequency synchronization. They showed (a) globally coupled identical phase oscillators cannot have weak chimera states and (b) there can be weak chimera state in some small networks of identical phase oscillators.

In this paper, we present some analysis and numerical investigations of weak chimera states in a particular system of six coupled identical phase oscillators. In Section IA we describe this network that was previously considered in [4]. Section II shows that this system of coupled phase oscillators has an integral of the motion for a particular value of coupling parameters. This is used in Theorem II.3 to show existence of an infinite number of weak chimeras for this integrable case. This result is rigorously proved in Section III by using integrability and a reversing symmetry to characterise motion in the phase plane.

In Section IV we show that these chimera states persist for nearby values of the parameters. We use the first return map to approximate these weak chimera solutions in the near-integrable limit. Although these are not analytically solvable, we are able to approximate the integrals numerically and compare them to numerical continuation results in order to understand stability and bifurcation of these weak chimera states. We finish with a discussion in Section V. 


\section{A. A minimal six oscillator network with weak chimeras}

Under sufficiently weak coupling, a system of $N$ coupled identical limit cycle oscillators can be reduced to a system of coupled identical phase oscillators [5], i.e. an ODE on the torus $\left(\theta_{1}, \ldots \theta_{N}\right) \in \mathbb{T}^{N}=[\mathbb{R} /(2 \pi \mathbb{Z})]^{N}$ of the form

$$
\dot{\theta}_{i}=\omega+\sum_{j=1}^{N} K_{i j} g\left(\theta_{i}-\theta_{j}\right)
$$

where $\omega$ is the natural frequency of the oscillators, $K_{i j}$ is the coupling strength from the $j$ th to the $i$ th oscillator and $g(\varphi)$ is a smooth $2 \pi$-periodic coupling or phase interaction function. If any oscillator can be replaced by any other, after a suitable relabelling we say these oscillators are indistinguishable [5].

The (angular) frequency $\Omega_{i}$ of oscillator $i$ in system (1) is the average rate of rotation of $\theta_{i}$, namely

$$
\Omega_{i}:=\lim _{T \rightarrow \infty} \frac{1}{T}\left[\theta_{i}(T)-\theta_{i}(0)\right] .
$$

Clearly this depends on the system and initial condition and indeed may not converge for some systems and initial conditions [8]. The frequency difference between oscillator $i$ and $j$ is defined as

$$
\Omega_{i j}:=\lim _{T \rightarrow \infty} \frac{1}{T}\left[\theta_{i}(T)-\theta_{j}(T)\right]=\Omega_{i}-\Omega_{j}
$$

We say there is frequency synchronization of oscillators $i$ and $j$ on a trajectory $\left(\theta_{1}(t), \theta_{2}(t), \ldots, \theta_{N}(t)\right)$ if $\Omega_{i j}=0$. We use the following definition of weak chimera state [4] (rather than a more general version in [8]).

Definition 1. A flow-invariant set $A \subset \mathbb{T}^{N}$ is a weak chimera state for a coupled $N$ phase oscillator system if it is connected, chain-recurrent and such that for each trajectory in $A$ there are oscillators $i, j$ and $k$ such that $\Omega_{i j} \neq 0$ and $\Omega_{i k}=0$ [4].

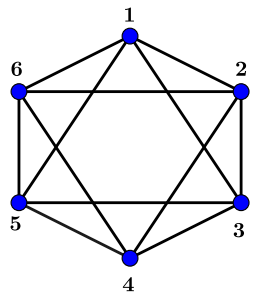

(A)

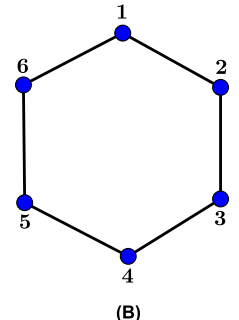

(B)

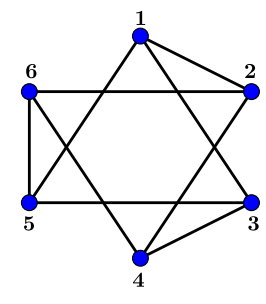

(C)
FIG. 1. Three networks of six indistinguishable oscillators that have weak chimera solutions for certain coupling: see [4]. We consider here the case (a) with non-global and non-local coupling which corresponds to (4). Of the other cases, (b) has local coupling while (c) has three inputs to each oscillator.
In [4] it was noted that all-to-all coupled (full permutation symmetric) networks of identical phase oscillators cannot have weak chimera solutions, but many networks of indistinguishable oscillators do: Figure 1 shows three coupling structures for six indistinguishable oscillators where weak chimera states are reported in [4].

This paper considers the system in Figure 1(a) with nearest neighbour and next-nearest neighbour coupling:

$$
\dot{\theta}_{i}=\omega+\sum_{|j-i|=1,2} g\left(\theta_{i}-\theta_{j}\right)
$$

with indexes taken module 6 , where $\theta_{i} \in[0,2 \pi)=\mathbb{T}$ denotes the phase oscillator, $\omega$ is the constant natural frequency of $\theta_{i}$. We use Hansel-Mato-Meunier coupling [13] with parameters $\alpha$ (or $\beta=\pi / 2-\alpha)[1,26]$ and $r$ :

$$
\begin{aligned}
g(\varphi) & :=-\sin (\varphi-\alpha)+r \sin (2 \varphi) \\
& =\cos (\varphi+\beta)+r \sin (2 \varphi) .
\end{aligned}
$$

We use $\beta$ rather than $\alpha$ because $r=\beta=0$ is an integrable limit. The study [4] found numerical evidence of stable and unstable weak chimeras in a particular invariant subspace $A_{1}$ that is parametrized by three independent phases:

$$
\left(\theta_{1}, \theta_{2}, \theta_{3}, \theta_{4}, \theta_{5}, \theta_{6}\right)=\left(\phi_{1}, \phi_{2}, \phi_{3}, \phi_{1}, \phi_{3}, \phi_{2}\right)
$$

We aim here to prove existence and understand stability of such states within $A_{1}$. Rewriting system (4) within $A_{1}$ in terms of $\left(\phi_{1}, \phi_{2}, \phi_{3}\right) \in \mathbb{T}^{3}$ gives

$$
\begin{aligned}
& \dot{\phi_{1}}=\omega+2 g\left(\phi_{1}-\phi_{2}\right)+2 g\left(\phi_{1}-\phi_{3}\right), \\
& \dot{\phi_{2}}=\omega+2 g\left(\phi_{2}-\phi_{1}\right)+g\left(\phi_{2}-\phi_{3}\right)+g(0), \\
& \dot{\phi_{3}}=\omega+2 g\left(\phi_{3}-\phi_{1}\right)+g\left(\phi_{3}-\phi_{2}\right)+g(0) .
\end{aligned}
$$

For convenience we introduce phase difference coordinates different to those in [4], that allow us to represent a time-reversal symmetry as a reflection. We define phase difference coordinates $(x, y) \in \mathbb{T}^{2}$ using $x=\phi_{1}-\left(\phi_{2}+\phi_{3}\right) / 2, y=\left(\phi_{2}-\phi_{3}\right) / 2$ so that $(7)$ can be written

$$
\begin{aligned}
\dot{x}= & 2[g(x-y)+g(x+y)]-g(-x-y) \\
& -g(0)-g(y-x)-[g(-2 y)+g(2 y)] / 2, \\
\dot{y}= & g(y-x)-g(-x-y)+[g(2 y)-g(-2 y)] / 2 .
\end{aligned}
$$

In the case $\beta=r=0$, note that $(5,8)$ reduces to

$$
\begin{aligned}
& \dot{x}=f(x, y)=2 \cos y \cos x-2 \cos ^{2} y \\
& \dot{y}=h(x, y)=2 \sin y \sin x
\end{aligned}
$$

Numerical simulation and continuation in [4] concluded there is a neutrally stable set of weak chimera states for this system that can be stabilized for $(\beta, r)$ near $(0,0)$. 


\section{INTEGRABILITY AND WEAK CHIMERA SOLUTIONS FOR A SYSTEM OF SIX OSCILLATORS}

In this section we first demonstrate there is an integrable structure in the subspace $A_{1}$ for (4) in the special case $r=\beta=0$ and use this to prove existence of weak chimeras. More precisely:

Lemma II.1. The system (4, 5) of six oscillators for $\beta=r=0$ has an integral of motion within the subspace $A_{1}$ defined by (6).

Proof. For the system on $A_{1}$ written as (9), we define

$$
E(x, y):=y+\cos y \sin y-2 \sin y \cos x .
$$

Taking partial derivatives of $E(x, y)$ with respect to both variables and using (9), the rate of change of $E$ on trajectories of (9) is

$$
\frac{d}{d t}[E(x(t), y(t))]=\frac{\partial E}{\partial x} \dot{x}+\frac{\partial E}{\partial y} \dot{y}=0,
$$

for all $x, y \in \mathbb{R}^{2}$. Hence $E(x, y)$ is constant on trajectories of (9) in this case: $E$ is an integral of the motion.

Figure 2 illustrates the level curves of $E(x, y)$ in the $(x, y)$ plane: each level curve is preserved by the dynamics of (9) and hence is a union of trajectories. We denote the level curve of $\tilde{E} \in \mathbb{R}$ by

$$
C(\tilde{E})=\left\{(x, y) \in \mathbb{T}^{2}: E(x, y)=\tilde{E}\right\} .
$$

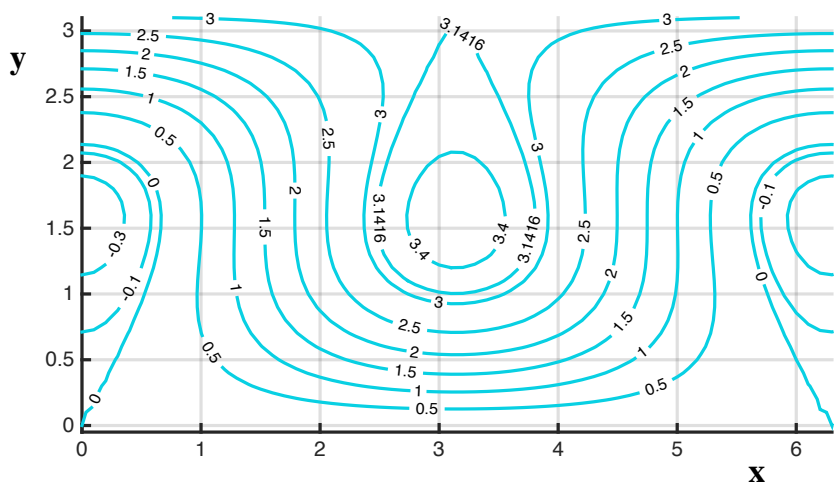

FIG. 2. The level curves for $E(x, y)$ defined by (10) in the region $(x, y) \in[0,2 \pi] \times[0, \pi]$. Each connected component of a level curve is invariant for the flow (9).

We consider the dynamics of (9) on the region

$$
M:=\{(x, y): 0<y<\pi \text { and } 0<x<2 \pi\}=H_{r} \cup H_{\ell},
$$

where $H_{r}:=\{(x, y): \pi<x<2 \pi$ and $0<y<\pi\}$ and $H_{\ell}:=\{(x, y): 0<x<\pi$ and $0<y<\pi\}$. Note that the upper and lower boundaries of $M$ are level curves for $E$ and hence invariant sets under the flow (see Figure 3).
The range of the integral of motion $E(x, y)$ for $(x, y) \in$ $\bar{M}$ comprises the interval $[\pi / 2-2, \pi / 2+2]$. The level curves $E(x, y)=\tilde{E}$ with $\tilde{E} \in(0, \pi)$ correspond to weak chimera states, while other level curves with $\tilde{E} \leq 0$ or $\tilde{E} \geq \pi$ correspond to frequency synchronized states. For almost all $\tilde{E} \in[0, \pi]$, the level curves in $M$ consist of periodic trajectories though there are exceptional level curves that contain equilibria and connecting orbits.

The system (9) has a reversing symmetry $R_{1}$ and a symmetry $R_{2}$, where

$$
R_{1}\left(\begin{array}{l}
x \\
y
\end{array}\right):=\left(\begin{array}{c}
2 \pi-x \\
y
\end{array}\right), \quad R_{2}\left(\begin{array}{l}
x \\
y
\end{array}\right):=\left(\begin{array}{c}
x \\
2 \pi-y
\end{array}\right),
$$

in the sense that if we write (9) as

$$
\left(\begin{array}{c}
\dot{x} \\
\dot{y}
\end{array}\right)=F\left(\begin{array}{l}
x \\
y
\end{array}\right)=\left(\begin{array}{c}
2 \cos y \cos x-2 \cos ^{2} y \\
2 \sin y \sin x
\end{array}\right)
$$

then

$$
F \circ R_{1}\left(\begin{array}{l}
x \\
y
\end{array}\right)=-R_{1} \circ F\left(\begin{array}{l}
x \\
y
\end{array}\right)
$$

and

$$
F \circ R_{2}\left(\begin{array}{l}
x \\
y
\end{array}\right)=R_{2} \circ F\left(\begin{array}{l}
x \\
y
\end{array}\right)
$$

for all $(x, y) \in \mathbb{T}^{2}$. A consequence of this is that the flow in $H_{r}$ corresponds to the time reversed flow on $R_{1}\left(H_{\ell}\right)$. We now state two main results that show existence of

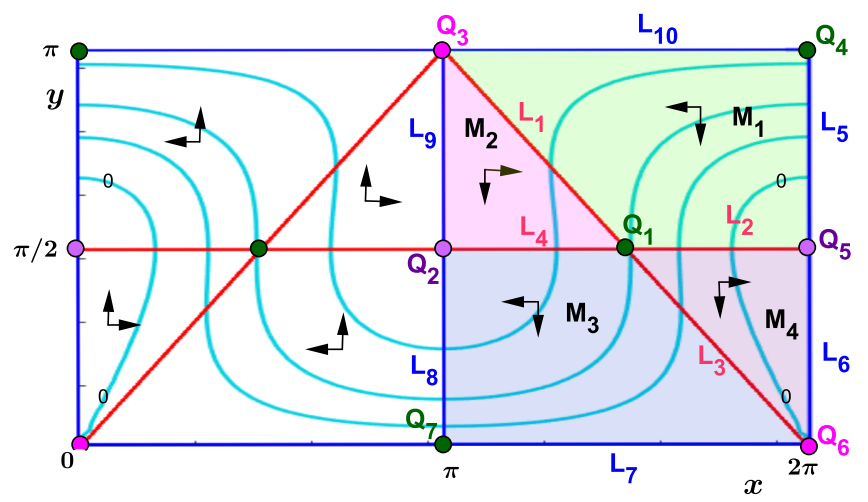

FIG. 3. The flow (9) on the invariant set $\bar{M} \subset \mathbb{T}^{2}$, where $M=\{(x, y): 0<y<\pi, 0<x<2 \pi\}$. The regions $H_{r}$ and $H_{\ell}=R_{1}\left(H_{r}\right)$ are shown as white and block of colored areas respectively. We define a partition of $H_{r}$ into a union of four open regions: green $M_{1}$, pink $M_{2}$, blue $M_{3}$, and purple $M_{4}$, points $Q_{i}, i=1,2,3,4,5,6,7$ and lines (without endpoints) $L_{i}, i=1,2, \ldots, 10$. The pink points $Q_{3}, Q_{6}$ are degenerate saddles while the purple points $Q_{2}, Q_{5}$ are centre equilibria. Trajectories are cyan, and black arrows represent direction of $\dot{x}, \dot{y}$. Blue/red lines are nullclines where $\dot{y}=0$ and $\dot{x}=0$ respectively. The nullclines allow us to decompose $M$ into a collection of open sets, in each of which the vector field points in one direction.

weak chimeras in the integrable case. 
Theorem II.2. Consider any $0<\tilde{E}<\pi$, then the level curve $C(\tilde{E})$ for system (9) is a trajectory $(x, y) \in \mathbb{R}^{2}$ and there is $T$ such that $x(T)=x(0)-2 \pi$ and $y(T)=y(0)$. More precisely, if $(x, y) \in C(\tilde{E})$ then

$$
\lim _{t \rightarrow \infty} \frac{1}{t} y(t)=0 \text { and } \lim _{t \rightarrow \infty} \frac{1}{t} x(t) \neq 0 .
$$

The proof of this is given in Section III C. A corollary of Theorem II.2 is the following result which gives existence of weak chimeras in this system.

Theorem II.3. The system $(4,5)$ of six oscillators with $\beta=r=0$ has an infinite number of chimera states within $A_{1}$ that are neutrally stable.

The proof of this is deferred to Section IIIC.

\section{DYNAMICS FOR THE INTEGRABLE CASE}

In order to understand properties of trajectories inside the set $M$, we partition the set into bounded areas using nullclines for system (9). We define the following subsets of $H_{r} \subset M$ (see Figure 3 ):

$$
\begin{aligned}
& M_{1}=\{(x, y): \pi / 2<y<\pi \text { and } 2 \pi-y<x<2 \pi\}, \\
& M_{2}=\{(x, y): \pi / 2<y<\pi \text { and } \pi<x<2 \pi-y\}, \\
& M_{3}=\{(x, y): 0<y<\pi / 2 \text { and } \pi<x<2 \pi-y\}, \\
& M_{4}=\{(x, y): 0<y<\pi / 2 \text { and } 2 \pi-y<x<2 \pi\} .
\end{aligned}
$$

We also define the following nullcline segments $L_{i}, i=$ $1,2, \ldots, 10$. (see Figure 3):

$$
\begin{aligned}
L_{1} & =\{(x, y): \pi / 2<y<\pi \text { and } x=2 \pi-y\}, \\
L_{2} & =\{(x, y): y=\pi / 2 \text { and } 3 \pi / 2<x<2 \pi\}, \\
L_{3} & =\{(x, y): 0<y<\pi / 2 \text { and } x=2 \pi-y\}, \\
L_{4} & =\{(x, y): y=\pi / 2 \text { and } \pi<x<3 \pi / 2\}, \\
L_{5} & =\{(x, y): \pi / 2<y<\pi \text { and } x=2 \pi\}, \\
L_{6} & =\{(x, y): 0<y<\pi / 2 \text { and } x=2 \pi\}, \\
L_{7} & =\{(x, y): y=0 \text { and } \pi<x<2 \pi\}, \\
L_{8} & =\{(x, y): 0<y<\pi / 2 \text { and } x=\pi\}, \\
L_{9} & =\{(x, y): \pi / 2<y<\pi \text { and } x=\pi\}, \\
L_{10} & =\{(x, y): y=\pi \text { and } \pi<x<2 \pi\} .
\end{aligned}
$$

Note that the boundary of each $M_{i}$ is a union of $\bar{L}_{i}$ as shown in Figure 3. Finally, we define the following points: $Q_{1}=(3 \pi / 2, \pi / 2), Q_{2}=(\pi, \pi / 2), Q_{3}=(\pi, \pi), Q_{4}=$ $(2 \pi, \pi), Q_{5}=(2 \pi, \pi / 2), Q_{6}=(2 \pi, 0)$ and $Q_{7}=(\pi, 0)$ (see Figure 3 ). Note that the only equilibria in $\bar{H}_{r}$ are $Q_{i}$, for $i=3,2,5,6$.
Lemma III.1. Note that $\bar{H}_{r}=\cup_{i=1}^{4} \bar{M}_{i}$, where the closures of the regions $M_{i}, i=1,2,3,4$ are as follows

$$
\begin{aligned}
& \bar{M}_{1}=L_{1} \cup L_{2} \cup L_{5} \cup L_{10} \cup Q_{1} \cup Q_{3} \cup Q_{4} \cup Q_{5} \cup M_{1}, \\
& \bar{M}_{2}=L_{1} \cup L_{4} \cup L_{9} \cup Q_{1} \cup Q_{3} \cup Q_{2} \cup M_{2}, \\
& \bar{M}_{3}=L_{4} \cup L_{8} \cup L_{3} \cup L_{7} \cup Q_{1} \cup Q_{2} \cup Q_{7} \cup Q_{6} \cup M_{3}, \\
& \bar{M}_{4}=L_{2} \cup L_{3} \cup L_{6} \cup Q_{1} \cup Q_{5} \cup Q_{6} \cup M_{4} .
\end{aligned}
$$

Proof. This proof is a verification that the sets define above have closures as stated.

\section{A. Dynamics of the integrable system on $M_{i}$}

We present a sequence of lemmas that analyse and describe the motion of trajectories on $H_{r} \subset M$. These lemmas are divided into three groups. The first two Lemmas III.2, III.3 identify the direction of trajectory inside the bounded areas $M_{1}, M_{2}, M_{3}$ and $M_{4}$. The second group gives conditions that implies the components of the solution of (9) are monotonic decreasing or increasing ( Lemma III.4). The third shows what happens when the trajectory reaches the boundary of each area after some finite time ( Lemmas III.5, III.6, III.7, III.8).

Lemma III.2. If $(x, y) \in M_{1}$ or $M_{3}$ then $f(x, y)<0$ and $h(x, y)<0$ for (9), (see Figure 3).

Proof. After factorizing (9), we note

$$
\begin{aligned}
& \dot{x}=2 \cos y(\cos x-\cos y), \\
& \dot{y}=2 \sin y \sin x .
\end{aligned}
$$

Suppose $(x, y) \in M_{1}$ then $\pi / 2<y<\pi$ and $2 \pi-y<$ $x<2 \pi$. Then, we get $\cos y<0$ and $\cos (2 \pi-y)<\cos x<$ $\cos (2 \pi)$, and simplifying, $\cos y<0$ and $0<\cos x-\cos y<$ $1-\cos y$. Because $\cos x$ is monotonic increasing on $x \in$ $(\pi, 2 \pi)$, then $\cos y<0$ and $\cos x-\cos y>0$ and we get that $f(x, y)<0$.

Also for the same point, we obtain $\sin y>0$ and $-\sin y<\sin x<0$ then $\sin y>0$ and $\sin x<0$ and we get that $h(x, y)<0$.

The statement for $M_{3}$ has a similar proof.

Lemma III.3. If $(x, y) \in M_{2}$ or $M_{4}$ then $f(x, y)>0$ and $h(x, y)<0$ for (12).

Proof. Suppose $(x, y) \in M_{2}$, this means $\pi / 2<y<\pi$ and $\pi<x<2 \pi-y$. Then, we get $\cos y<0$ and $\cos (\pi)<$ $\cos x<\cos (2 \pi-y)$, This is the same $\cos y<0$ and $-1-\cos y<\cos x-\cos y<0$ which implies that $f(x, y)>$ 0 .

Following that, at the same point $(x, y) \in M_{2}$, we get $\sin y>0$ and $-\sin y<\sin x<0$. Hence $\sin y>0$ and $\sin x<0$ which implies that $h(x, y)<0$.

The statement for $M_{4}$ has a similar proof.

In the next lemma, we prove that the solution of (9) monotonically decreases or monotonically increases along the trajectory within a region such as $M_{i}$. 
Lemma III.4. Suppose $\mathcal{R}$ is a connected region in $\mathbb{R}^{2}$ such that $f(x, y)$ and $h(x, y)$ have no zero in $\mathcal{R}$. Suppose $(x(t), y(t))$ is a solution of $\dot{x}=f(x, y), \dot{y}=h(x, y)$ such that $(x(t), y(t)) \in \mathcal{R}$ for $0<t<T$. The following holds

(i) if $f(x, y)<0$ and $h(x, y)<0$, then $x(t)$ and $y(t)$ are monotonically decreasing with $t$ for $0<t<T$.

(ii) if $f(x, y)>0$ and $h(x, y)<0$, then $x(t)$ is monotonically increasing and $y(t)$ is monotonically decreasing for $0<t<T$.

Proof. We begin by proving (i). From the system (9), we consider the first component

$$
\frac{d x}{d t}=f(x, y)
$$

Consider any $0<t_{0}<t_{1}<T$ and integrate both sides with respect to $t$ we get,

$$
\int_{t_{0}}^{t_{1}} \frac{d x}{d t}(t) d t=\int_{t_{0}}^{t_{1}} f(x(t), y(t)) d t
$$

for all $0<t_{0}<t_{1}<T$. Hence $x\left(t_{1}\right)-x\left(t_{0}\right)=$ $\int_{t_{0}}^{t_{1}} f(x(t), y(t)) d t$. Since $f(x(t), y(t))<0$ for all $t_{0}<$ $t<t_{1}$, then $x\left(t_{1}\right)-x\left(t_{0}\right)=\int_{t_{0}}^{t_{1}} f(x(t), y(t)) d t<0$. Then $x\left(t_{1}\right)<x\left(t_{0}\right)$ is monotonically decreasing for all $0<t_{0}<t_{1}<T$.

Using similar steps and the assumption $h(x, y)<0$ we prove $y\left(t_{1}\right)<y\left(t_{0}\right)$ is also monotonically decreasing for all $0<t_{0}<t_{1}<T$.

The proof for (ii) follows similarly.

The following Lemmas III.5, III.6, III.7 and III.8 explain what happens for an initial condition after time $T$ for (9).

Lemma III.5. If an initial condition $(x(0), y(0)) \in M_{1} \cup$ $L_{5}$, then there exist $T>0$ such that $(x(T), y(T)) \in L_{1} \cup$ $L_{2} \cup Q_{1}$.

Proof. Suppose $(x(0), y(0)) \in M_{1}$, then $x(t)$ and $y(t)$ are monotonically decreasing as long as they remain in $M_{1}$ (Lemma III.4(i)). Either there exists $T>0$ such that $(x(T), y(T)) \in \overline{M_{1}} \backslash M_{1}$ or $(x(t), y(t)) \in M_{1}$ for all $t>0$. In the first case there are two possibilities to describe the path of trajectory. Firstly, if the initial point $(x(0), y(0)) \in M_{1}$ then the trajectory of this point will eventually hit the boundary of $M_{1}$ at $\left(L_{1} \cup L_{2} \cup Q_{1}\right)$. Secondly, if the initial point $(x(0), y(0)) \in L_{5}$ then we still have $f(x(0), y(0))<0$ so $x(t)$ is monotonically decreasing for initial condition on $L_{5}$. Here for arbitrary small $t>0$, $(x(t), y(t)) \in M_{1}$ and we apply the argument above.

In the second case, let the greatest lower bound of $x(t)$ and $y(t)$ be $x_{\infty}$ and $y_{\infty}$ respectively, (according to theorem of limits of monotone functions [14]). We get that

$$
\lim _{t \rightarrow \infty}(x(t), y(t))=\left(x_{\infty}, y_{\infty}\right) .
$$

Then $\left(x_{\infty}, y_{\infty}\right)$ is a limit point. Since a limit set is an invariant set which has a single point then it is an equilibrium point [27]. Note there are no equilibrium points in $M_{1}$ so $\left(x_{\infty}, y_{\infty}\right) \in \bar{M}_{1} \backslash M_{1}$. By monotonicity for $(x(t), y(t))$, it would be in $L_{1} \cup L_{2} \cup Q_{1}$ which is a contradiction because no equilibrium point belongs to $L_{1} \cup L_{2} \cup Q_{1}$. As a consequence, the second case can not happen.

Lemma III.6. If an initial condition $(x(0), y(0)) \in M_{2} \cup$ $L_{1}$, then there exist $T>0$ such that $(x(T), y(T)) \in L_{4}$.

Proof. Suppose $(x(0), y(0)) \in M_{2}$, then $x(t)$ is monotonically increasing and $y(t)$ is monotonically decreasing as long they remain in $M_{2}$ (Lemma III.4(ii)). Either there exists $T>0$ such that $(x(T), y(T)) \in \overline{M_{2}} \backslash M_{2}$ or $(x(t), y(t))$ stay in $M_{2}$ for all $t>0$. In the first case there are two possibilities. Firstly, if the initial point $(x(0), y(0)) \in M_{2}$ then the trajectory of $(x(0), y(0))$ will eventually hit the boundary of $M_{2}$ at $L_{4}$. Secondly, if the initial point $(x(0), y(0)) \in L_{1}$ then we still have $h(x, y)<0$ so $y(t)$ is still monotonically decreasing for initial condition on $L_{1}$. Here for arbitrary small $t>0$, $(x(t), y(t)) \in M_{2}$ and we apply the argument above.

In the second case, let the least upper bound $x(t)$ and the greatest lower bound $y(t)$ be $x_{\infty}$ and $y_{\infty}$ respectively, (according to theorem of limits of monotone functions [14]). We get

$$
\lim _{t \rightarrow \infty}(x(t), y(t))=\left(x_{\infty}, y_{\infty}\right) .
$$

Then $\left(x_{\infty}, y_{\infty}\right)$ is an equilibrium point [27] and $\left(x_{\infty}, y_{\infty}\right) \in \overline{M_{2}}$. Note that there is no equilibrium point in $M_{2}$ so $\left(x_{\infty}, y_{\infty}\right) \in \overline{M_{2}} \backslash M_{2}$. By monotonicity for $(x(t), y(t))$, it would be in $L_{4}$ which is a contradiction because no equilibrium point belongs to $L_{4}$. As a consequence, the second case cannot happen.

Lemma III.7. If $(x(0), y(0)) \in M_{4} \cup L_{2}$ then either there exists $T>0$ such that $(x(T), y(T)) \in L_{3} \cup L_{6}$ or $\lim _{t \rightarrow \infty}(x(t), y(t))=Q_{6}$.

Proof. Suppose $(x(0), y(0)) \in M_{2}$ then $x(t)$ is monotonically increasing and $y(t)$ is monotonically decreasing as long they remain in $M_{4}$ (Lemma III.4(ii)). Either there exists $T>0$ such that $(x(T), y(T)) \in \overline{M_{4}} \backslash M_{4}$ or it stays in $M_{4}$ for all $T>0$. In the first case there are two possibilities. Firstly, if the initial point $(x(0), y(0)) \in M_{4}$ then the trajectory of $(x(0), y(0))$ will eventually hit the boundary of $M_{4}$ at $L_{3} \cup Q_{6} \cup L_{6}$. As $Q_{6}$ is an equilibrium point, we can only hit the boundary at $L_{3} \cup L_{6}$. Secondly, if the initial point $(x(0), y(0)) \in L_{2}$ then we still have $h(x, y)<0$ so $y(t)$ is still monotonically decreasing for initial condition on $L_{2}$. Here for arbitrary small $t>0$, $(x(t), y(t)) \in M_{4}$ and we apply the argument above. In the second case, monotonicity [14] implies that

$$
\lim _{t \rightarrow \infty}(x(t), y(t))=\left(x_{\infty}, y_{\infty}\right) .
$$

Thus, $x_{\infty}$ is the least upper bound of $x(t)$ and $y_{\infty}$ is the greatest lower bound of $y(t)$. This means that 
$(x(t), y(t)) \rightarrow\left(x_{\infty}, y_{\infty}\right)$ is a limit point and then it is an equilibrium point as $t \rightarrow \infty$ [27]. Since $Q_{6}$ is the only equilibrium point in $\bar{M}_{4}$ we have that $\left(x_{\infty}, y_{\infty}\right)=$ $Q_{6}$.

Lemma III.8. If ( $x(0), y(0)) \in M_{3} \cup L_{4} \cup L_{3} \cup Q_{1}$, then there exists $T>0$ such that $(x(T), y(T)) \in L_{8}$.

Proof. Suppose $(x(0), y(0)) \in M_{2}$, then $x(t)$ is monotonically increasing and $y(t)$ is monotonically decreasing as long they remain in $M_{3}$ (Lemma III.4(i)). Either there exists $T>0$ such that $(x(T), y(T)) \in \bar{M}_{3} \backslash M_{3}$ or they stay in $M_{3}$ for all $T>0$. In the first case there are two possibilities. Firstly, if the initial point $(x(0), y(0)) \in M_{3}$ then the trajectory of the of $(x(0), y(0))$ will eventually hit the boundary of $M_{3}$ at $L_{8} \cup L_{7} \cup Q_{7}$. However $(x(T), y(T)) \in L_{7} \cup Q_{7}$ as $y=0$ because it is invariant under the flow. Hence if it hits the boundary after finite time, it can only hit $L_{8}$. Secondly, if the initial point $(x(0), y(0)) \in L_{4}, L_{3}$ or $Q_{1}$ then we still have $h(x, y)<0$ so $y(t)$ is still monotonically decreasing for initial condition on $L_{4}, L_{3}$ and $Q_{1}$. Here for arbitrary small $t>0$, $(x(t), y(t)) \in M_{3}$ and we apply the argument above.

In the second case, let the greatest lower bound of $x(t)$ and $y(t)$ be $x_{\infty}$ and $y_{\infty}$ respectively, (according to theorem of limits of monotone functions [14]). We get that

$$
\lim _{t \rightarrow \infty}(x(t), y(t))=\left(x_{\infty}, y_{\infty}\right) .
$$

Then $\left(x_{\infty}, y_{\infty}\right)$ is limit point. Since a limit set is an invariant set that is a single point then it is an equilibrium point [27]. Note there are no equilibrium points in $M_{3}$ so $\left(x_{\infty}, y_{\infty}\right) \in \overline{M_{3}} \backslash M_{3}$. By monotonicity for $(x(t), y(t))$, it would be in $L_{8}$ which is a contradiction because no equilibrium points belong to $L_{8}$. As a consequence, the second case can not happen.

\section{B. Extending trajectories from $H_{r}$ to $H_{\ell}$}

In this section, we discuss the structure of trajectories on the set $M$. The first lemma gives a condition that implies existence of a periodic orbit that winds in the first component $(x)$ of torus.

Lemma III.9. For any $0<\tilde{E}<\pi$ there is an initial condition $(x(0), y(0)) \in C(\tilde{E})$ and $T>0$ such that if $(x(t), y(t))$ is a trajectory of the system (9) then $x(T)=$ $x(0)-2 \pi$ and $y(T)=y(0)$.

Proof. Let $0<\tilde{E}<\pi$. We claim there is an $(x(0), y(0)) \in$ $C(\tilde{E}) \cap L_{5}$. To see this, we consider $(x(0), y(0))=(2 \pi, z)$, $z \in(\pi / 2, \pi)$ which parametrizes $L_{5}$. Note that

$$
E(2 \pi, z)=-2+\pi / 2<0 \text { for } z=\pi / 2,
$$

and

$$
E(2 \pi, z)=\pi \text { for } z=\pi \text {. }
$$

Hence for any $\tilde{E} \in(0, \pi)$ there exists $z \in(\pi / 2, \pi)$ such that $E(2 \pi, z)=\tilde{E}$ by the Intermediate Value Theorem. Indeed, from (10) one can show that $E(2 \pi, z)$, is a monotonically increasing with $z \in[\pi / 2, \pi]$, and hence there is a unique $z$ such that $E(2 \pi, z)=\tilde{E}$. Suppose $(x(t), y(t))$ is the trajectory of (9) that starts at $(x(0), y(0)) \in$ $C(\tilde{E}) \cap L_{5}$. According to Lemma III.5, there exists $T_{1}>0$ such that $\left(x\left(T_{1}\right), y\left(T_{1}\right)\right) \in L_{1} \cup Q_{1} \cup L_{2}$. We have the following

1. If $\left(x\left(T_{1}\right), y\left(T_{1}\right)\right) \in L_{1}$ then by Lemma III.6 there exists $T_{2}>T_{1}$ such that $\left(x\left(T_{2}\right), y\left(T_{2}\right)\right) \in L_{4}$, and then by Lemma III.8, there exists $T_{3}>T_{2}$ such that $\left(x\left(T_{3}\right), y\left(T_{3}\right)\right) \in L_{8}$.

2. if $\left(x\left(T_{1}\right), y\left(T_{1}\right)\right) \in Q_{1}$ then by Lemma III.8, there exists $T_{3}>T_{1}$ such that $\left(x\left(T_{3}\right), y\left(T_{3}\right)\right) \in L_{8}$.

3. If $\left(x\left(T_{1}\right), y\left(T_{1}\right)\right) \in L_{2}$ then by Lemma III.7 and using the fact $\tilde{E} \leq 0$ on $L_{6} \cup Q_{6}$ there exists $T_{2}>T_{1}$ such that $\left(x\left(T_{2}\right), y\left(T_{2}\right)\right) \in L_{3}$. According to Lemma III.8, there exists $T_{3}>T_{2}$ such that $\left(x\left(T_{3}\right), y\left(T_{3}\right)\right) \in L_{8}$.

We now show that this trajectory reaches $x(T)=$ $x(0)-2 \pi, y(T)=y(0)$ at $T:=2 T_{3}$. Firstly, we have defined $H_{r}$ and $H_{\ell}$, such that $R_{1}\left(H_{r}\right)=H_{\ell}$. Let the trajectory $(x(t), y(t)) \in H_{r}$ for all $0<t<T_{3}$ and we define $(\tilde{x}(t), \tilde{y}(t)):=R_{1}(x(t), y(t))$. Because $R_{1}$ is a reversing symmetry on the torus, note that $((\tilde{x}(-t), \tilde{y}(-t))$ is also a trajectory. Moreover, we note that $R_{1}\left(L_{8}\right)=L_{8}$ and define

$$
\begin{aligned}
\tilde{L}_{5} & :=R_{1}\left(L_{5}\right)=\{(2 \pi-x, y): \pi / 2<y<\pi, x=2 \pi\} \\
& =\{(0, y): \pi / 2<y<\pi\} .
\end{aligned}
$$

Note that $(x(0), y(0)) \in L_{5} \cap C(\tilde{E})$ and $\left(x\left(T_{3}\right), y\left(T_{3}\right)\right) \in$ $L_{8}$. Now $(\tilde{x}(-t), \tilde{y}(-t))$ is a trajectory on $H_{\ell}$ for $-T_{3}<$ $t<0$. Also,

$$
(\tilde{x}(0), \tilde{y}(0))=R_{1}(x(0), y(0))=(2 \pi-x(0), y(0)) \in \tilde{L}_{5},
$$

and

$\left(\tilde{x}\left(T_{3}\right), \tilde{y}\left(T_{3}\right)\right)=R_{1}\left(x\left(T_{3}\right), y\left(T_{3}\right)\right)=\left(x\left(T_{3}\right), y\left(T_{3}\right)\right)=L_{8}$.

So if we define $\left(x\left(T_{3}+t\right), y\left(T_{3}+t\right)\right)=\left(\tilde{x}\left(T_{3}-t\right), \tilde{y}\left(T_{3}-\right.\right.$ $t)), 0<t<T_{3}$ then $(x(t), y(t))$ is a trajectory for $0<t<$ $2 T_{3}$. In particular, we have shown there is a trajectory such that $x(T)=x(0)-2 \pi$ and $y(T)=y(0)$.

Recall that the $\omega$-limit set $\Lambda^{+}(x, y)$, and the $\alpha$-limit set $\Lambda^{-}(x, y)$ are defined:

$$
\begin{aligned}
& \Lambda^{+}(x, y):=\bigcap_{T>0} \overline{\bigcup_{s>T} \varphi(x, y, s)}, \\
& \Lambda^{-}(x, y):=\bigcap_{T<0} \overline{\bigcup_{s<T} \varphi(x, y, s)}
\end{aligned}
$$

where $\varphi(x, y, s)$ is the flow generated by $(9)$. 
Lemma III.10. Suppose $\tilde{E}=0$, then there is a point $(x(0), y(0)) \in C(\tilde{E}) \cap L_{5}$ such that $(x(t), y(t))$ is a homoclinic orbit that limits to $Q_{6}$ (see Figure 2).

Proof. For $\tilde{E}=0$, we claim that there is an initial condition $(x(0), y(0)) \in C(\tilde{E}) \cap L_{5}$ such that $E(x(0), y(0))=0$. Consider $(x(0), y(0))=(2 \pi, z)$ where $z \in(\pi / 2, \pi)$. By a similar argument to the proof of Lemma III.9 there exists $z \in(\pi / 2, \pi)$ such that $E(2 \pi, z)=\tilde{E}$. If we write $(x(t), y(t))$ to be the solution of system (9) starting at the point $(x(0), y(0))$ then $E(x(t), y(t))=0$ for all $t>0$. According to Lemma III.5, there is $T_{1}>0$ such that the trajectory of $(x(t), y(t))$ will hit $L_{2}$. Depending on Lemma III.7, either there is $T_{2}>T_{1}$ such that $\left(x\left(T_{2}\right), y\left(T_{2}\right)\right) \in L_{3} \cup L_{6}$ or $(x(t), y(t)) \in M_{4}$ stays at $M_{4}$ for all $t>0$ and converges to $Q_{6}$ as $t \rightarrow \infty$. We will focus just on the second possibility. Because the system (9) is reversible then there is $T_{2}<0$ such that the trajectory of $(x(-t), y(-t))$ converges to $Q_{6}$ for all $0>t>-T_{2}$. More precisely, we have

$$
\lim _{t \rightarrow \pm \infty} E(x(t), y(t))=Q_{6},
$$

so that $\Lambda^{+}(x, y)=\Lambda^{-}(x, y)=Q_{6}$ This trajectory is called a homoclinic orbit [22].

\section{Proof of existence of weak chimeras}

In this section we provide proofs of Theorems II.2 and II.3: namely the existence of an infinite number of chimera states that are neutrally stable for the integrable case (9).

Proof. (Theorem II.2) Define $Y_{0}=\{(x, 0): 0 \leq x \leq 2 \pi\}$ and $Y_{\pi}=\{(x, \pi): 0 \leq x \leq 2 \pi\}$ as the lower and upper boundaries of $M$. Note that $Y_{0}$ and $Y_{\pi} \subset \bar{M}$. These boundaries are invariant sets for the system (9) since $\dot{y}=$ 0 for all $Y_{0}$ and $Y_{\pi}$, therefore

$$
0 \leq y(t) \leq \pi
$$

for all $t>0$ and

$$
0 \leq \frac{y(t)}{t} \leq \frac{\pi}{t}, \quad \forall t>0
$$

We now take the limit and obtain

$$
0 \leq \lim _{t \rightarrow \infty} \frac{1}{t}[y(t)] \leq \lim _{t \rightarrow \infty} \frac{\pi}{t} .
$$

In consequence, $\lim _{t \rightarrow \infty} \frac{1}{t}[y(t)]=0$ for any $0<\tilde{E}<\pi$ and $(2 \pi, y) \in C(\tilde{E})$.

Now we will prove the second part of the result: Let $(x(0), y(0)) \in C(\tilde{E})$ such that $(x(0), y(0))=(2 \pi, y(0))$ by Lemma III.9 there is a $0<T<\infty$ such that $(x(T), y(T))=(0, y(0))$. If we define $n=n(t):=\lfloor t / T\rfloor$, where $\lfloor t / T\rfloor$ denotes the largest integer number less than or equal $t / T$, then

$$
n T \leq t<(n+1) T
$$

and hence

$$
-2 \pi n<x(t) \leq-2 \pi(n-1) .
$$

Dividing (14) by (13), we get that

$$
\frac{-2 \pi n}{(n+1) T}>\frac{x(t)}{t}>\frac{-2 \pi(n-1)}{n T} .
$$

By taking the limit for (15), we get

$$
-\frac{2 \pi}{T} \geq \lim _{t \rightarrow \infty} \frac{x(t)}{t} \geq-\frac{2 \pi}{T},
$$

which can be simplified as follows

$$
\lim _{t \rightarrow \infty} \frac{x(t)}{t}=-\frac{2 \pi}{T} \neq 0 .
$$

Proof. (Theorem II.3) We know that $y=\frac{1}{2}\left(\phi_{2}-\phi_{3}\right)$ and $x=\phi_{1}-\phi_{3}-y$. Also, from Theorem II.2 with given hypothesis, we have that for any $0<\tilde{E}<\pi$ there is $t$ such that

$$
\lim _{t \rightarrow \infty} \frac{1}{t} y(t)=0,
$$

which is expanded to

$$
\lim _{t \rightarrow \infty} \frac{1}{t} y(t)=\frac{1}{2} \lim _{t \rightarrow \infty} \frac{1}{t}\left[\phi_{2}(t)-\phi_{3}(t)\right]=\frac{1}{2} \Omega_{23} .
$$

Then from (16) and (17), we obtain

$$
\frac{1}{2} \Omega_{23}=\lim _{t \rightarrow \infty} \frac{1}{t}[y(t)]=0 .
$$

This means there is frequency synchronization between $\phi_{2}(T)$ and $\phi_{3}(T)$. To show that $\phi_{1}$ and $\phi_{3}$ are not frequency synchronized $\Omega_{13} \neq 0$. We need to prove that a curve from $x=0$ to $x=2 \pi$ is continuous and there is no an equilibrium point on the level curve. Let $(x(0), y(0)) \in C(\tilde{E})$ and $0<\tilde{E}<\pi$ be an initial condition for the system (9). Then there is $T>0$ such that $(x(T), y(T))=(x(0)-2 \pi, y(0))$ (Lemma III.9), (i.e. the trajectory will return to its initial condition after time $T$ ). In addition, the solution for this initial condition satisfies $\lim _{t \rightarrow \infty} \frac{1}{t}(x(t), y(t))=(-2 \pi / T, 0) \neq(0,0)$, (Theorem II.3 ). That means this trajectory does not converge to a equilibrium point (Lemma III.10) and continuous. According to Theorem II.2 we have

$$
\lim _{t \rightarrow \infty} \frac{1}{t} x(t)=-\frac{2 \pi}{T}<0 .
$$

which implies that $\Omega_{13} \neq 0$. This means there is frequency desynchronization between $\phi_{1}$ and $\phi_{3}$. From above argument, we get a weak chimera solution (Definition 1). As a consequence, for each $\tilde{E}$ in the infinite set $(0, \pi)$ there is a distinct chimera state. As these chimera periodic orbit are not isolated they are not hyperbolic. 


\section{EXISTENCE AND STABILITY OF WEAK CHIMERAS FOR $(\beta, r) \neq(0,0)$}

In this section we extend the analysis of the integrable case $(\beta, r)=(0,0)$ to nearby $(\beta, r)$ for the planar system (8). We use perturbation techniques and the fact that (10) is slowly varying to describe existence, stability and bifurcation of weak chimera solutions for this regime and compare with results from numerical simulations and continuation in [4].

In Section IV A, we compute the period and other properties for chimera solutions with $\beta, r=0$. In Section IV B approximates chimera solutions $\beta, r \neq 0$. Finally, Section IV C analyses bifurcation of chimera states for this approximation and compare to the numerical results.

\section{A. Properties of integrable weak chimeras}

We write $(5,8)$ as the following system for $(x, y) \in \mathbb{T}^{2}$ depending on two parameters $\beta$ and $r$,

$$
\begin{aligned}
\dot{x} & =24 r \sin x \cos x \cos ^{2} y-6 \sin x \cos y \sin \beta \\
& +2 \cos x \cos y \cos \beta-12 r \sin x \cos x \\
& -2 \cos \beta \cos ^{2} y, \\
\dot{y} & =2 \sin y\left(4 r \cos ^{2} x \cos y+4 r \cos ^{3} y+\sin x \cos \beta\right. \\
& -\cos x \sin \beta-\cos y \sin \beta-4 r \cos y) .
\end{aligned}
$$

The above system has an integral of motion for $\beta=r=$ 0 : see Lemma II.1 and $E(x, y)$ as in (10).

In order to describe the stability of weak chimera solutions for $\beta, r \neq 0$ and any level curve $0<\tilde{E}<\pi$ we first need to understand properties of the level curves $C(\tilde{E})$ for $0<\tilde{E}<\pi$. As can be seen in Figure 3, the level curves are not graphs over $x$ or $y$ for the whole period $T \in(0,2 \pi)$. Therefore, we highlight the region $H_{r}$ from $x(0)=2 \pi k$ to $x(T / 2)=2 \pi k-\pi$. The geometric properties of all the level curves on half period $H_{r}$ is that each of these levels pass through maxima of $y$ at $\left(2 \pi k, y_{+}(\tilde{E})\right)$ to minima of $y$ at $\left(2 \pi k-\pi, y_{-}(\tilde{E})\right)$ for $k \in \mathbb{N}$. In particular, in this range of $\tilde{E}$, the curve $(x, y) \in C(\tilde{E}) \cap H_{r}$ is a graph over

$$
y_{-}(\tilde{E}) \leq y \leq y_{+}(\tilde{E})
$$

given by the unique solution of $E(x, y)=\tilde{E}$ with $x \in$ $[\pi, 2 \pi]$. This can be written

$$
x=\Delta_{\tilde{E}}(y):=2 \pi-\arccos \left[\frac{\cos y \sin y+y-\tilde{E}}{2 \sin y}\right]
$$

where arccos is the usual inverse on $[0, \pi]$. To find $y_{+}(\tilde{E})$ and $y_{-}(\tilde{E})$ we substitute $x(0)=2 \pi$ and $x(T / 2)=\pi$ in (10) and solve for $y$. Maple's fsolve is used to give numerical approximations of $y_{+}(\tilde{E})$ and $y_{-}(\tilde{E})$.
The period $T(\tilde{E})$ of the trajectory on the level curve $0<\tilde{E}<\pi$ is found for the system (9). We eliminate $x$ by substituting (20) in the equation for $\dot{y}$ of (9) to give

$$
\dot{y}=h\left(\Delta_{E_{0}}(y), y\right)=-\sqrt{4 \sin ^{2} y-(\cos y \sin y+y-\tilde{E})^{2}} .
$$

If we integrate this over the half period within $H_{r}$ we obtain

$$
\frac{T(\tilde{E})}{2}=-\int_{t=0}^{\frac{T(\tilde{E})}{2}} \frac{1}{\sqrt{4 \sin y^{2}-(\cos y \sin y+y-\tilde{E})}} d y .
$$

Writing the integration limits in terms of $y$ then gives

$$
T(\tilde{E})=2 \int_{y_{-}(\tilde{E})}^{y_{+}(\tilde{E})} \frac{1}{\sqrt{4 \sin y^{2}-(\cos y \sin y+y-\tilde{E})}} d y
$$

Figure 4 shows numerical approximation of $T(\tilde{E})$ for $\tilde{E} \in(0, \pi)$, computed using Maple's fsolve. Note that $T(\tilde{E}) \rightarrow \infty$ as $\tilde{E} \rightarrow 0+$ or $\tilde{E} \rightarrow \pi-$. There is a symmetry $T(\tilde{E})=T(-\tilde{E}+\pi)$, and $T(\tilde{E})$ has a unique minimum at $\tilde{E}=\pi / 2$.

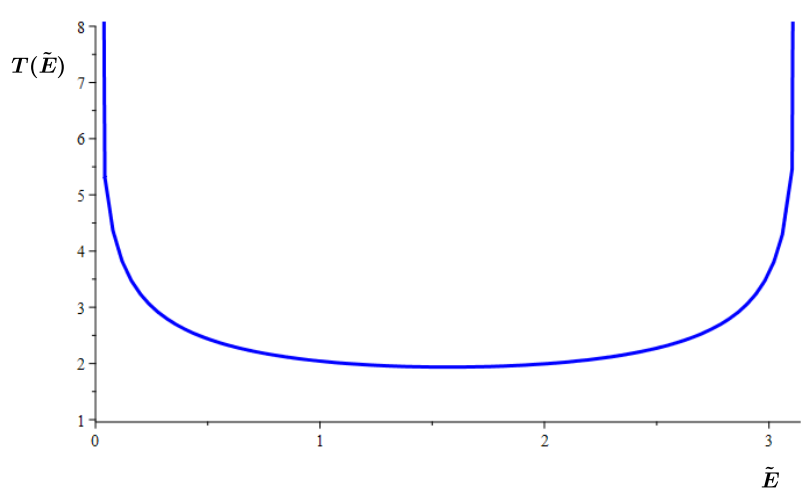

FIG. 4. Period $T(\tilde{E})$ of the weak chimera solution of (9) for $\tilde{E} \in(0, \pi)$ in the integrable case $\beta=r=0$, computed using (21). Observe that the period tends to infinity as the level curve approaches the homoclinic orbits at $\tilde{E}=0$ and $\pi$.

\section{B. Approximation of chimeras for $(\beta, r) \neq(0,0)$}

The aim of this section is to find an approximate Poincaré map for the more general case (18) and use this map to prove existence of chimera states for $\beta, r \neq 0$.

We choose a Poincaré section $\Sigma_{p} \subseteq \mathbb{T}^{2}$ where

$$
\Sigma_{p}=\left\{(x, y) \in \mathbb{T}^{2}: x=2 \pi \text { and } y \in(0, \pi)\right\} .
$$

The time of first return of $(x, y) \in \Sigma_{p}$ is defined by

$$
\tau(x, y):=\inf \left\{t>0: \varphi(x, y, t) \in \Sigma_{p} \text { and }(x, y) \in \Sigma_{p}\right\}
$$

where $\varphi(x, y, t)$ is the flow of the system (18). The Poincaré first return map $P: \Sigma_{p} \rightarrow \Sigma_{p}$ is then defined 
by $P(x, y)=\varphi(x, y, \tau(x, y))$. Let us write $P(2 \pi, y)=$ $P\left(2 \pi, y_{+}(\tilde{E})\right)$ and parametrize the chimera using $\tilde{E} \in$ $(0, \pi)$. Then $P(2 \pi, y)=P\left(2 \pi, y_{+}(\tilde{E})\right)=\left(2 \pi, y_{+}(\tilde{P}(\tilde{E}))\right)$. So the first return $P$ can be understood as a map $\tilde{P}$ : $(0, \pi) \rightarrow(0, \pi)$,

$$
E_{n+1}=\tilde{P}\left(E_{n}\right) .
$$

We know in the integrable case, the chimera periodic orbits intersect $\Sigma_{p}$ at fixed points of the Poincaré map, i.e $\tilde{P}(\tilde{E})=\tilde{E}$ for all $\tilde{E}$ and $\beta=r=0$. More generally, note that

$$
\frac{d}{d t}[E(x(t), y(t))]=\frac{\partial E}{\partial x} \dot{x}+\frac{\partial E}{\partial y} \dot{y}
$$

for all $(x, y) \in \mathbb{R}^{2}$. Combining (18) and (23) we get

$$
\frac{d E}{d t}(x, y)=G(x, y) \sin \beta+F(x, y) r,
$$

where

$$
\begin{aligned}
G(x, y) & :=4 \sin y\left[4 \cos y \cos ^{2} x-\cos ^{3} y-3 \cos y\right], \\
F(x, y) & :=4 \sin y\left[4 \cos ^{5} y-4 \cos ^{4} y \cos x\right. \\
& +4 \cos ^{3} y \cos ^{2} x-16 \cos ^{2} y \cos ^{3} x-4 \cos ^{3} y \\
& \left.+16 \cos ^{2} y \cos x+6 \cos ^{3} x-6 \cos x\right] .
\end{aligned}
$$

Substituting (20) in (24) and defining $G_{\tilde{E}}(y)$ := $G\left(\Delta_{\tilde{E}}(y), y\right)$ and $F_{\tilde{E}}(y):=F\left(\Delta_{\tilde{E}}(y), y\right)$ we get

$$
\frac{d \tilde{E}}{d t}=\frac{d E}{d t}\left(\Delta_{\tilde{E}}(y), y\right)=G_{\tilde{E}}(y) \sin \beta+F_{\tilde{E}}(y) r .
$$

Now assume that $(\beta, r)=(\epsilon \tilde{\beta}, \epsilon \tilde{r})$ and consider the behaviour of (18) in the limit $0<\epsilon \ll 1$ for fixed $(\tilde{\beta}, \tilde{r}) \neq(0,0)$. Note

$$
\frac{d E}{d t}\left(\Delta_{\tilde{E}}(y), y\right)=\epsilon\left[G_{\tilde{E}}(y) \tilde{\beta}+F_{\tilde{E}}(y) \tilde{r}\right],
$$

and so on trajectories $(x(t), y(t)), \tilde{E}(t)$ varies slowly with $t$ if $\epsilon$ is small.

Suppose now that $E_{0} \in(0, \pi)$ and $(x(0), y(0)) \in \Sigma_{p} \cap$ $C\left(E_{0}\right)$. For any $T>0$ such that $(x(t), y(t)) \in H_{r}$ for all $0<t<T$, we can approximate (20) to get

$$
x(t)=\Delta_{\tilde{E}(t)}(y(t))=\Delta_{E_{0}}(y(t))+O(\epsilon),
$$

where the error term also depends linearly on $t$. Moreover, if $T\left(E_{0}\right)<\infty$ then (using the symmetry between $H_{r}$ and $H_{\ell}$ ) there will be first return to $\Sigma_{p}$ after time $T\left(E_{0}\right)+O(\epsilon)$ that is close to $E_{0}$.

We approximate this first return (22) in the case $\epsilon>0$ : for $(x(t), y(t)) \in H_{r}$, note that using (27) in (26) we have

$$
\frac{d E}{d t}\left(\Delta_{\tilde{E}}(y), y\right)=\epsilon\left[G_{E_{0}}(y) \tilde{\beta}+F_{E_{0}}(y) \tilde{r}\right]+O\left(\epsilon^{2}\right) .
$$

If the trajectory next intersects $\Sigma_{p}$ at $\left(0, y_{+}\left(E_{1}\right)\right)$ after time $T=T\left(E_{0}\right)+O(\epsilon)$ then we can approximate

$$
\begin{aligned}
E_{1}-E_{0} & =\int_{t=0}^{T} \frac{d E}{d t} d t \\
& =2 \epsilon \int_{t=0}^{\frac{T\left(E_{0}\right)}{2}}\left[G_{E_{0}}(y) \tilde{\beta}+F_{E_{0}}(y) \tilde{r}\right] d t+O\left(\epsilon^{2}\right) \\
& =\epsilon \Lambda\left(E_{0}, \tilde{\beta}, \tilde{r}\right)+O\left(\epsilon^{2}\right) .
\end{aligned}
$$

where (changing the limits of integration) and defining $h_{E_{0}}(y):=h\left(\Delta_{E_{0}}(y), y\right)$ we can write

$$
\begin{aligned}
\Lambda\left(E_{0}, \tilde{\beta}, \tilde{r}\right) & :=-2 \int_{y_{-}\left(E_{0}\right)}^{y_{+}\left(E_{0}\right)} \frac{G_{E_{0}}(y) \tilde{\beta}+F_{E_{0}}(y) \tilde{r}}{h_{E_{0}}(y)} d y \\
& =-2\left(\Lambda_{1}\left(E_{0}\right) \tilde{\beta}+\Lambda_{2}\left(E_{0}\right) \tilde{r}\right),
\end{aligned}
$$

where

$$
\begin{aligned}
& \Lambda_{1}\left(E_{0}\right):=\int_{y_{-}\left(E_{0}\right)}^{y_{+}\left(E_{0}\right)} \frac{G_{E_{0}}(y)}{h_{E_{0}}(y)} d y, \\
& \Lambda_{2}\left(E_{0}\right):=\int_{y_{-}\left(E_{0}\right)}^{y_{+}\left(E_{0}\right)} \frac{F_{E_{0}}(y)}{h_{E_{0}}(y)} d y .
\end{aligned}
$$

Hence the Poincaré map $E_{1}=\tilde{P}\left(E_{0}\right)$ satisfies

$$
\begin{aligned}
\tilde{P}\left(E_{0}\right) & =E_{0}+\epsilon \Lambda\left(E_{0}, \tilde{\beta}, \tilde{r}\right)+O\left(\epsilon^{2}\right) \\
& =E_{0}-2 \epsilon\left[\Lambda_{1}\left(E_{0}\right) \tilde{\beta}+\Lambda_{2}\left(E_{0}\right) \tilde{r}\right]+O\left(\epsilon^{2}\right) .
\end{aligned}
$$

In the next theorem, we show the existence of a symmetric weak chimera state for $\Lambda\left(E_{n}, \tilde{\beta}, \tilde{r}\right)$ at $E_{0}=\pi / 2$ and $(\beta, r) \neq(0,0)$.

Theorem IV.1. For almost all $(\tilde{\beta}, \tilde{r})$, if $\epsilon$ is small enough then system (18) with $(\beta, r)=(\epsilon \tilde{\beta}, \epsilon \tilde{r})$ has a periodic orbit that is close to the level curve of (10) corresponding to $E_{0}=\pi / 2$. This periodic orbit corresponds to a weak chimera state of (30).

Proof. Note that in the special case $E_{0}=\pi / 2$, (30) gives

$$
\Lambda(\pi / 2, \tilde{\beta}, \tilde{r})=-2\left[\Lambda_{1}(\pi / 2) \tilde{\beta}+\Lambda_{2}(\pi / 2) \tilde{r}\right] .
$$

In this case, $y_{+}=\pi-y_{-}$can be found from (19) and (10) using $E_{0}=\pi / 2$ to give

$$
\begin{aligned}
& \pi / 2=y_{+}+\cos y_{+} \sin y_{+}-2 \sin y_{+}, \\
& \pi / 2=\pi-y_{+}-\cos y_{+} \sin y_{+}+2 \sin y_{+} .
\end{aligned}
$$

Hence $y_{+}=\pi-y_{-}$for $E=\pi / 2$. We now compute

$$
\begin{aligned}
& \Lambda_{1}(\pi / 2)=\int_{y_{-}}^{\pi / 2} \hat{G}(y) d y+\int_{\pi / 2}^{y_{+}} \hat{G}(y) d y, \\
& \Lambda_{2}(\pi / 2)=\int_{y_{-}}^{\pi / 2} \hat{F}(y) d y+\int_{\pi / 2}^{y_{+}} \hat{F}(y) d y,
\end{aligned}
$$

where we define

$$
\hat{G}(y)=\frac{G_{\pi / 2}(y)}{h_{\pi / 2}(y)}, \quad \hat{F}(y)=\frac{F_{\pi / 2}(y)}{h_{\pi / 2}(y)} .
$$


Because $\hat{G}(y)=-\hat{G}(\pi-y)$ and $\hat{F}(y)=-\hat{F}(\pi-y)$ we can compute

$$
\Lambda_{1}(\pi / 2)=\Lambda_{2}(\pi / 2)=0 .
$$

Hence, $\Lambda(\pi / 2, \tilde{\beta}, \tilde{r})=0$ for all $\tilde{\beta}$, and $\tilde{r}$. On the other hand, one can check numerically that neither of $\Lambda_{1}^{\prime}(\pi / 2)$ or $\Lambda_{2}^{\prime}(\pi / 2)$ are zero, which means that for every choice of $\tilde{\beta}, \tilde{r}$ such that

$$
\Lambda_{1}^{\prime}(\pi / 2) \tilde{\beta}+\Lambda_{2}^{\prime}(\pi / 2) \tilde{r} \neq 0 .
$$

We approximate the derivative by using small $h$ in

$$
\Lambda_{1}^{\prime}(\pi / 2) \approx \frac{\Lambda_{1}(\pi / 2+h)-\Lambda_{1}(\pi / 2)}{h},
$$

and

$$
\Lambda_{2}^{\prime}(\pi / 2) \approx \frac{\Lambda_{2}(\pi / 2+h)-\Lambda_{2}(\pi / 2)}{h} .
$$

Using $h=0.0001$, we obtain $\Lambda_{1}^{\prime}(\pi / 2)=10.1374$ and $\Lambda_{2}^{\prime}(\pi / 2)=16.1335$. Hence the derivative $\Lambda^{\prime}(\pi / 2, \tilde{\beta}, \tilde{r})$ is non-zero for almost all $\tilde{\beta}, \tilde{r}$. For these cases and small enough $\epsilon$ the Poincaré map $\tilde{P}$ will have a hyperbolic fixed point that limits to $E_{0}=\pi / 2$ as $\epsilon \rightarrow 0$. The flow trajectory corresponding to this fixed point is a periodic orbit will remain close to the contour $C(\pi / 2)$ : hence there is a chimera state for small enough $\epsilon>0$.

Note that if (34) is not satisfied this implies that $\tilde{\beta} \approx$ $-1.5914 \tilde{r}$ which corresponds to a pitchfork bifurcation of chimera states: this is discussed in the next Section.

\section{Bifurcation of weak chimera solutions}

It is well known that if the Poincaré map $\tilde{P}$ given in (31) has a fixed point $E^{*}$ then the fixed point will be stable if $\left|\tilde{P}^{\prime}\left(E^{*}\right)\right|<1$ (resp. unstable if $\left.\left|\tilde{P}^{\prime}\left(E^{*}\right)\right|>1\right)$ ( see e.g. [21, Theorem 9.3]). Because

$$
\tilde{P}^{\prime}\left(E_{0}\right)=1+\epsilon \Lambda^{\prime}\left(E_{0}, \tilde{\beta}, \tilde{r}\right)+O\left(\epsilon^{2}\right)
$$

(and the error term is uniform in any closed subinterval of $\left.E_{0} \in(0, \pi)\right)$ it follows that if there is an $E_{0} \in(0, \pi)$ such that $\Lambda\left(E_{0}, \tilde{\beta}, \tilde{r}\right)=0$ and $\Lambda^{\prime}\left(E_{0}, \tilde{\beta}, \tilde{r}\right)<0$ (resp. $\left.\Lambda^{\prime}\left(E_{0}, \tilde{\beta}, \tilde{r}\right)>0\right)$ then there is a stable (resp. unstable) chimera periodic orbit near $C\left(E_{0}\right)$ for small enough $\epsilon>$ 0 . This means that bifurcations condition of chimera periodic orbits near $\epsilon=0$ correspond to a nonhyperbolic fixed point $E_{0}$ such that $\Lambda\left(E_{0}, \beta, \tilde{r}\right)=\Lambda^{\prime}\left(E_{0}, \tilde{\beta}, \tilde{r}\right)=0$.

Figure 5 shows $\Lambda\left(E_{0}, \tilde{\beta}, \tilde{r}\right)$ on varying $E_{0}$ for a range of $\tilde{\beta} \in(-1,1)$ and fixed $\tilde{r}=-0.01$ computed using (30) and Maple's numerical integration evalf/int. The zeros of this clearly show the location of nontrivial zeros $\Lambda\left(E_{0}, \tilde{\beta}, \tilde{r}\right)=0$ that may be stable (negative slope) or unstable (positive slope) in the limit of small $\epsilon$.
We also compute an asymptotic bifurcation diagram by numerically locating the zero contour

$$
\Lambda\left(E_{0}, \tilde{\beta}, \tilde{r}\right)=0
$$

on varying $\tilde{\beta}$ and $E_{0}$ for fixed $\tilde{r}$, using Maple's fsolve. This is shown in Figure 6: the lower case letters and index of $i=1, \ldots, 5$ refer to fixed points in Figure 5. Bifurcation points in Figure 6 can be identified as three types: there are saddle-node, pitchfork and homoclinic bifurcations that occur (for $\tilde{r}<0$ at parameter values $\tilde{\beta}_{S n}<\tilde{\beta}_{P f}<\tilde{\beta}_{H m}$ respectively in the limit $\epsilon \rightarrow+0$.

The scenario for a saddle-node bifurcation has three stages: there is only one stable point $b_{1}$ for $\tilde{\beta}<\tilde{\beta}_{S n}$. A saddle-node or tangent bifurcation of symmetrically placed fixed points for the Poincaré map at $\tilde{\beta}=\tilde{\beta}_{S n}$ creates degenerate fixed points $a_{2}, c_{2}$ (red curve for Figure 5) with $\left|P^{\prime}\left(E_{a_{2}}\right)\right|=\left|P^{\prime}\left(E_{c_{2}}\right)\right|=1$. At these points (where the red curve is tangent to the $E$-axis) the qualitative behaviour of the system becomes structurally unstable, in that an arbitrarily small perturbation leads to a change of the qualitative dynamics of system. Finally, for $\tilde{\beta}_{S n}<\tilde{\beta}<\tilde{\beta}_{P f}$, two pairs of fixed points (unstable and stable) $a_{1}, a_{3}$ and $c_{1}, c_{3}$ appear as zeros of the green curve.

The pairs of fixed points $a_{1}$ and $c_{1}$ collide with $b_{1}$ on the cyan line at $\tilde{\beta}=\beta_{P f}$ where the pitchfork bifurcation occurs. This is where the fixed point $b_{1}$ is marginally stable, $\left|P^{\prime}\left(E_{b_{1}}\right)\right|=1$ and $\Lambda^{\prime}\left(E_{b_{1}}, \tilde{\beta}, \tilde{r}\right)=0$. For $\tilde{\beta}_{P f}<$ $\tilde{\beta}$ there are three fixed points (for example, $a_{5}, c_{5}$ and $b_{1}$ where the orange curve has zeros. The point $b_{1}$ is unstable while $a_{5}$ and $c_{5}$ are stable.

The two fixed points $a_{6}$ and $c_{6}$ hit the boundary of the domain $E \in[0, \pi]$ at the homoclinic bifurcation where $\tilde{\beta}_{H_{m}}=\tilde{\beta}$. At this point the period of the weak chimera solution becomes infinite in the limit: see Figure 4 and the magenta line for Figure 5. At the homoclinic bifurcation the points $a_{7}$ and $c_{7}$ move to the boundary and for $\tilde{\beta}_{H_{m}}<\tilde{\beta}$ the only remaining fixed point is at $b_{1}$. A similar scenario of bifurcations is found for $\tilde{r}>0$ though with the stabilities reversed: there is a parameter-time reversing symmetry given by $(\beta, r, t) \mapsto(-\beta,-r,-t)$.

Asymptotic approximations of the bifurcations from (30) are compared to continuation results for the original system (18) on varying $\beta$, for $r=-0.01$ using XPPAUT as in [4]: see Figure 6 and Table I. We find good agreement for saddle-node and pitchfork bifurcations of weak chimeras from the asymptotic theory and from numerical continuation respectively, while the homoclinic bifurcation seems to have errors that grow faster with $\epsilon$. This is not too surprising, as we expect the approximation errors to be larger in the high period limit. Figure 7 summarises and compares the bifurcations of chimeras for (30) and (18) in the parameter space $(\beta, r)$. 


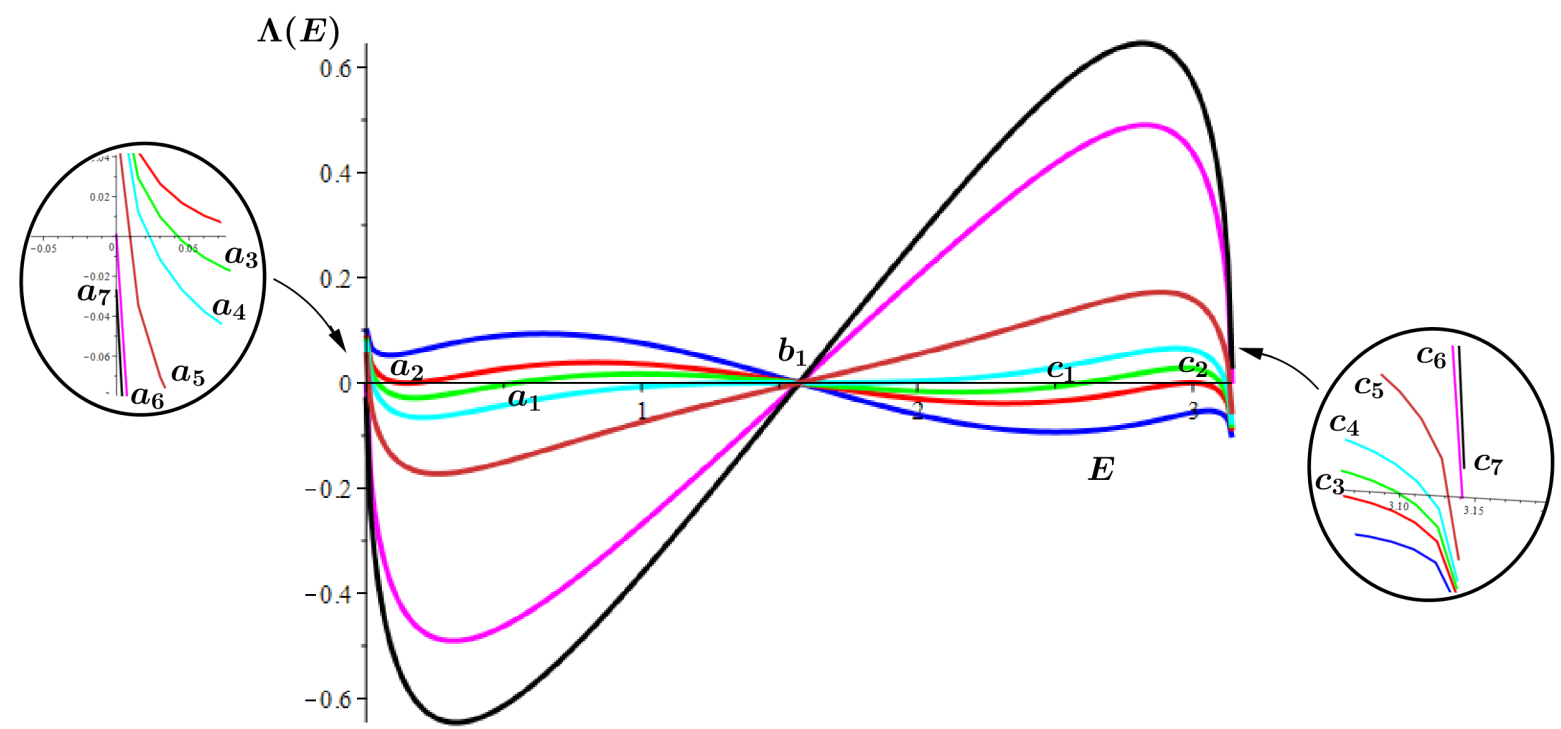

FIG. 5. Approximations of (30) for various values of $\tilde{\beta}$ and $\tilde{r}=-0.01$, where zeros of $\Lambda(E)=-2\left[\Lambda_{1}(E) \tilde{\beta}+\Lambda_{2}(E) \tilde{r}\right]$ correspond to fixed points of the approximate Poincaré map. The points $b_{1}$ and $a_{i}, c_{i}, i=1, . ., 6$ represent various fixed points on varying $\tilde{\beta}$. The coloured curves (blue, red, green, cyan, orange, magenta and black) correspond to $\tilde{\beta}=$ $0.0062,0.0081,0.0125,0.0159,0.028,0.063$ and 0.08 respectively. The red curve is at the saddle-node bifurcation while the cyan curves is at the pitchfork bifurcation. The homoclinic bifurcation can be seen for the magenta curve.

\begin{tabular}{|c|c|c|}
\hline Type of Bif. & $\beta$ using (30) & $\beta$ using (18) \\
\hline Sn of LC & 0.0081 & 0.0081 \\
\hline Pf of LC & 0.0159 & 0.0158 \\
\hline Hom of LC & 0.063 & 0.0192 \\
\hline
\end{tabular}

TABLE I. Comparison of the approximations of $\beta$ at saddle node of limit cycle, pitchform and homoclinic bifurcations of weak chimeras for $r=-0.01$. The first column gives the asymptotic approximate system (30) for the limit $\epsilon \rightarrow 0$. The second column gives values from continuation of (18) using XPPAUT. Observe there is very good agreement for the first two bifurcations while the third only gives better agreement closer to the integrable limit: see Figure 7.

\section{DISCUSSION}

For a non-locally coupled network of six identical and indistinguishable oscillators with nearest neighbour and next-nearest neighbour coupling $(4,5)$ and a particular value of the coupling parameters $r=\beta=0$, we prove existence of an infinite number of neutrally stable weak chimera states. We examine nullclines of the system (9) and use a reversing symmetry in this integral case to understand the structure of phase space. We use nullclines for the system (9) and a reversing symmetry is used to prove a sequence of lemmas on the structure of phase space. This is used in Theorem II.3 to prove the existence of infinitely many neutrally stable weak chimeras.

In this paper we restrict from the full system (4 of six phases) (5) to a two dimensional system of phase differences in a three dimensional invariant subspace (8).
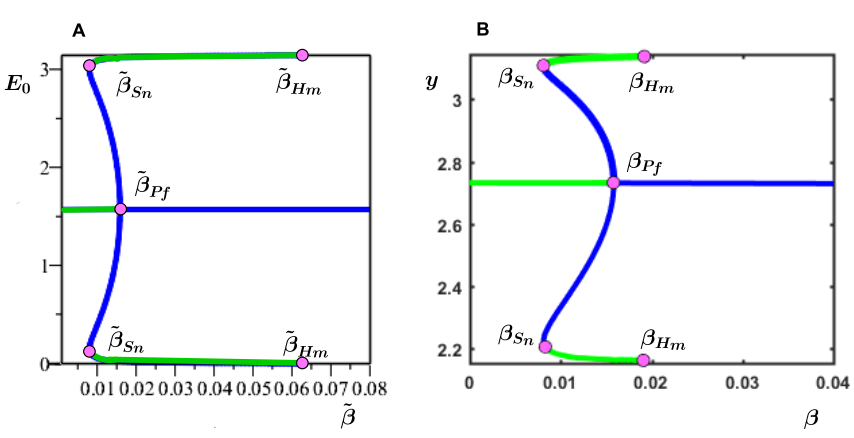

FIG. 6. Left panel (A) shows bifurcation digram ( $\tilde{\beta}$ against E) for system (30) when $\tilde{r}=-0.01$ (numerically approximated by Maple for the limit $\epsilon \rightarrow 0)$, while right panel $(B)$ shows bifurcation diagram ( $\beta$ against $y$ ) for system (18) and $r=-0.01$ (numerically approximated by XPPAUT). Blue and green lines denote unstable/stable periodic orbit. In both Panels $A$ and $B$, there is saddle-node bifurcation for limit cycle at points $\tilde{\beta}_{S n}$ and $\beta_{S n}$ and pitchfork at points $\tilde{\beta}_{P f}$ and $\beta_{P f}$. The points $\tilde{\beta}_{H m}$ and $\beta_{H m}$ indicate homoclinic bifurcation.

The reduced system (9) has an integrable structure in the special case when $r=\beta=0$ which means every level curve of the phase portrait has a specific value. We have not yet determined the stability of any of the solutions to perturbations away from the invariant subspace, but this should be possible, at least numerically.

On changing the parameters $r$ and $\beta$ we continue and find bifurcations of certain weak chimeras. Section IV considers weakly dissipative perturbations of the inte- 


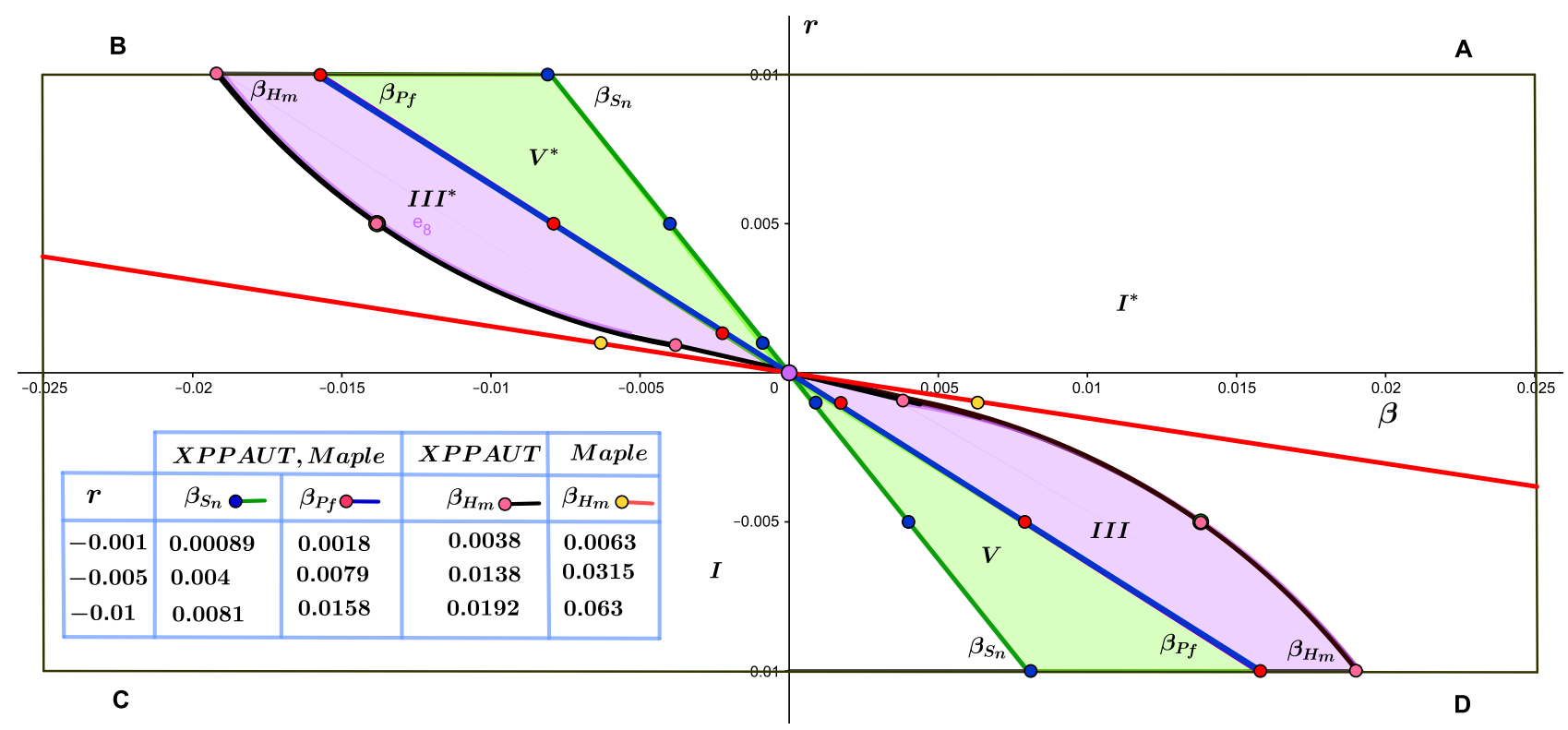

FIG. 7. Bifurcation diagrams for the asymptotic approximation (30) computed with Maple and the contination (18) computed with XPPAUT in the parameter space $(\beta, r)$ close to the integral case $(0,0)$. Bifurcations for (18) are computed varying $\beta$ for $r= \pm 0.001, \pm 0.005$ and \pm 0.01 : these are shown as coloured points. Bifurcation lines for (30) are computed using Maple and represented by the blue and green lines showing $(\beta, r)=\epsilon(\tilde{\beta}, \tilde{r})$ for $\epsilon \in \mathbb{R}$. Note that quadrants $A$ (resp. B) and $C$ (resp. D) have the same qualitative dynamics on reversing time because of the time-reversing parameter symmetry $(\beta, r, t) \mapsto(-\beta,-r,-t)$. There are four coloured regions (green and purple) where multiple chimera states coexist in the asymptotic limit. There is only one chimera within the white regions: this is stable in $I$ and unstable in $I^{*}$. Five branches of chimera (three stable and two unstable) are in the green area $V$, (also, two stable and three unstable at $V^{*}$ ), bounded by a saddle node of limit cycles (green line) and a pitchfork of limit cycles (blue line). There are two stable chimera and one unstable in purple area III, (also, one stable and two unstable at III*), bounded by the pitchfork and the homoclinic bifurcation (black curve) that are only present in the XPPAUT calculations, (homoclinic bifurcation: the red line is computed using Maple).

grable system with $(\beta, r)=(0,0)$. By constructing an approximate first return map (31) we are able to continue certain weak chimera states for sufficiently small change of parameters. We note that depending on the ratio of $\beta / r$, the number of chimeras and their type changes as we approach the integrable limit (see Figure 6). These computations still require computation of integrals that we approximate numerically: it would clearly be of interest to find a completely analytical explanation of these bifurcations.

Although it is not clear which of these methods will generalize to more complex and higher dimensional systems with weak chimeras, but it seems very likely that considering the dynamics of systems near $\beta=r=0$ as weakly dissipative perturbations of a degenerate system with integrals of the motion (as here and in [26]) will still be helpful.

\section{Acknowledgements}

We thank the Iraqi Ministry of Higher Education and Scientific Research (MOHESR) for support of MT via a Scholarship. We thank Yuri Maistrenko, Jan Sieber and Chris Bick for very interesting discussions about aspects of this research.
[1] Daniel M Abrams, Rennie Mirollo, Steven H Strogatz, and Daniel A Wiley. Solvable model for chimera states of coupled oscillators. Physical Review Letters, 101(8):084103, (2008).

[2] Daniel M Abrams and Steven H Strogatz. Chimera states for coupled oscillators. Physical Review Letters, 93(17):174102, (2004).

[3] Daniel M Abrams and Steven H Strogatz. Chimera states in a ring of nonlocally coupled oscillators. International Journal of Bifurcation and Chaos, 16(01):21-37, (2006).
[4] Peter Ashwin and Oleksandr Burylko. Weak chimeras in minimal networks of coupled phase oscillators. Chaos: An Interdisciplinary Journal of Nonlinear Science, 25(1):013106, (2015).

[5] Peter Ashwin and James W Swift. The dynamics of $\mathrm{n}$ weakly coupled identical oscillators. Journal of Nonlinear Science, 2(1):69-108, (1992).

[6] Tanmoy Banerjee and Debarati Ghosh. Transition from amplitude to oscillation death under mean-field diffusive coupling. Physical Review E, 89(5):052912, (2014). 
[7] Bidesh K Bera, Dibakar Ghosh, and M Lakshmanan. Chimera states in bursting neurons. Physical Review E, 93(1):012205, (2016).

[8] Christian Bick and Peter Ashwin. Chaotic weak chimeras and their persistence in coupled populations of phase oscillators. Nonlinearity, 29(5):1468, (2016).

[9] Qionglin Dai, Mengya Zhang, Hongyan Cheng, Haihong Li, Fagen Xie, and Junzhong Yang. From collective oscillation to chimera state in a nonlocally coupled excitable system. Nonlinear Dynamics, pages 1-9, (2018).

[10] Lucia Valentina Gambuzza, Arturo Buscarino, Sergio Chessari, Luigi Fortuna, Riccardo Meucci, and Mattia Frasca. Experimental investigation of chimera states with quiescent and synchronous domains in coupled electronic oscillators. Physical Review E, 90(3):032905, (2014).

[11] R Gopal, VK Chandrasekar, A Venkatesan, and M Lakshmanan. Observation and characterization of chimera states in coupled dynamical systems with nonlocal coupling. Physical Review E, 89(5):052914, (2014).

[12] Aaron M Hagerstrom, Thomas E Murphy, Rajarshi Roy, Philipp Hövel, Iryna Omelchenko, and Eckehard Schöll. Experimental observation of chimeras in coupled-map lattices. Nature Physics, 8(9):658, (2012).

[13] D Hansel, G Mato, and C Meunier. Clustering and slow switching in globally coupled phase oscillators. Physical Review E, 48(5):3470, (1993).

[14] John K Hunter. An introduction to real analysis. University of California at Davis, California, (2012).

[15] Patrycja Jaros, Serhiy Brezetsky, Roman Levchenko, Dawid Dudkowski, Tomasz Kapitaniak, and Yuri Maistrenko. Solitary states for coupled oscillators with inertia. Chaos: An Interdisciplinary Journal of Nonlinear Science, 28(1):011103, (2018).

[16] Y. Kuramoto and D. Battogtokh. Coexistence of coherence and incoherence in nonlocally coupled phase oscillators. Nonlinear Phenom. Complex Syst. 5, 380, (2002).

[17] Yoshiki Kuramoto. Chemical waves. In Chemical Oscillations, Waves, and Turbulence, pages 89-110. Springer, (1984).

[18] Carlo R Laing. Chimeras in networks of planar oscillators. Physical Review E, 81(6):066221, (2010).

[19] Carlo R Laing. Chimeras in networks with purely local coupling. Physical Review E, 92(5):050904, (2015).

[20] Carlo R Laing and Carson C Chow. Stationary bumps in networks of spiking neurons. Neural Computation, 13(7):1473-1494, (2001).

[21] GC Layek. An Introduction to Dynamical Systems and Chaos. Springer, (2015).

[22] Stephen Lynch. Dynamical systems with applications using Mathematica. Springer Science \& Business Media, (2007).

[23] Erik Andreas Martens, Shashi Thutupalli, Antoine Fourrière, and Oskar Hallatschek. Chimera states in mechanical oscillator networks. Proceedings of the National Academy of Sciences, 110(26):10563-10567, (2013).

[24] E Omelchenko, Yuri L Maistrenko, and Peter A Tass. Chimera states: The natural link between coherence and incoherence. Physical Review Letters, 100(4):044105, (2008).

[25] Iryna Omelchenko, Anna Zakharova, Philipp Hövel, Julien Siebert, and Eckehard Schöll. Nonlinearity of local dynamics promotes multi-chimeras. Chaos: An Interdisciplinary Journal of Nonlinear Science, 25(8):083104, (2015).
[26] Mark J Panaggio, Daniel M Abrams, Peter Ashwin, and Carlo R Laing. Chimera states in networks of phase oscillators: the case of two small populations. Physical Review E, 93(1):012218, (2016).

[27] Lawrence Perko. Differential equations and dynamical systems, volume 7. Springer Science \& Business Media, (2013).

[28] K Premalatha, VK Chandrasekar, M Senthilvelan, and M Lakshmanan. Impact of symmetry breaking in networks of globally coupled oscillators. Physical Review E, 91(5):052915, (2015).

[29] Lennart Schmidt, Konrad Schönleber, Katharina Krischer, and Vladimir García-Morales. Coexistence of synchrony and incoherence in oscillatory media under nonlinear global coupling. Chaos: An Interdisciplinary Journal of Nonlinear Science, 24(1):013102, (2014).

[30] Gautam C Sethia and Abhijit Sen. Chimera states: the existence criteria revisited. Physical Review Letters, 112(14):144101, (2014).

[31] Gautam C Sethia, Abhijit Sen, and Fatihcan M Atay. Clustered chimera states in delay-coupled oscillator systems. Physical Review Letters, 100(14):144102, (2008).

[32] Jan Sieber, E Omelchenko, and Matthias Wolfrum. Controlling unstable chaos: stabilizing chimera states by feedback. Physical Review Letters, 112(5):054102, (2014).

[33] Mark R Tinsley, Simbarashe Nkomo, and Kenneth Showalter. Chimera and phase-cluster states in populations of coupled chemical oscillators. Nature Physics, 8(9):662, (2012).

[34] Matthias Wolfrum and E Omelchenko. Chimera states are chaotic transients. Physical Review E, 84(1):015201, (2011).

[35] Matthias Wolfrum, Oleh E Omel'chenko, and Jan Sieber. Regular and irregular patterns of self-localized excitation in arrays of coupled phase oscillators. Chaos: An Interdisciplinary Journal of Nonlinear Science, 25(5):053113, (2015). 